\title{
Daily walnut intake improves metabolic syndrome status and increases circulating adiponectin levels: randomized controlled crossover trial
}

\author{
Hyo-Jeong Hwang', Yanan Liu' ${ }^{2}$, Hyun-Sook Kim², Heeseung Lee ${ }^{3}$, Yunsook Lim ${ }^{3}$ and Hyunjin Park ${ }^{15}$ \\ 'ICAN Nutrition Education and Research, 20, Gukjegeumyung-ro 7-gil, Yeongdeungpo-gu, Seoul 07340, Republic of Korea \\ ${ }^{2}$ Department of Food and Nutrition, Sookmyung Women's University, Seoul 04310, Republic of Korea \\ ${ }^{3}$ Department of Food and Nutrition, Kyung Hee University, Seoul 02447, Republic of Korea
}

BACKGROUND/OBJECTIVES: Several previous studies have investigated whether regular walnut consumption positively changes heart-health-related parameters. The aim of this study was to investigate the effects of daily walnut intake on metabolic syndrome (MetS) status and other metabolic parameters among subjects with MetS.

SUBJECTS/METHODS: This study was a two-arm, randomized, controlled crossover study with 16 weeks of each intervention (45 g of walnuts or iso-caloric white bread) with a 6 week washout period between interventions. Korean adults with MetS $(n=119)$ were randomly assigned to one of two sequences; 84 subjects completed the trial. At each clinic visit (at 0 , 16, 22, and 38 weeks), MetS components, metabolic parameters including lipid profile, hemoglobin A1c (HbA1c), adiponectin, leptin, and apolipoprotein $\mathrm{B}$, as well as anthropometric and bioimpedance data were obtained.

RESULTS: Daily walnut consumption for 16 weeks improved MetS status, resulting in $28.6 \%-52.8 \%$ reversion rates for individual MetS components and $51.2 \%$ of participants with MetS at baseline reverted to a normal status after the walnut intervention. Significant improvements after walnut intake, compared to control intervention, in high-density lipoprotein cholesterol (HDL-C) $(P=0.028)$, fasting glucose $(P=0.013), \mathrm{HbA1c}(P=0.021)$, and adiponectin $(P=0.019)$ were observed after adjustment for gender, age, body mass index, and sequence using a linear mixed model.

CONCLUSION: A dietary supplement of $45 \mathrm{~g}$ of walnuts for 16 weeks favorably changed MetS status by increasing the concentration of HDL-C and decreasing fasting glucose level. Furthermore, consuming walnuts on a daily basis changed HbA1c and circulating adiponectin levels among the subjects with MetS. This trial is registered at ClinicalTrials.gov as NCT03267901.

Nutrition Research and Practice 2019;13(2):105-114; https://doi.org/10.4162/nrp.2019.13.2.105; plSSN 1976-1457 elSSN 2005-6168

Keywords: Metabolic syndrome, juglans, adiponectin, cross-over studies

\section{INTRODUCTION}

Metabolic syndrome (MetS), which is comprised of central obesity, elevated blood pressure, increased levels of fasting blood glucose and triglycerides (TG), and decreased high-density lipoprotein cholesterol (HDL-C), has been well-established as a risk factor for type 2 diabetes (T2D) and cardiovascular disease [1]. Due to rapid transitions toward high energy intake and sedentary lifestyle in the last few decades, MetS has become a major health challenge globally. In South Korea, $31.3 \%$ of Korean adults have been affected according to the revised National Cholesterol Education Program (NCEP) Adult Treatment Panel III [2]. In particular, the percentage of the population with hypercholesterolemia has been gradually increasing. Prevalence of hypercholesterolemia among Koreans aged 30 and above has increased from $8.0 \%$ in 2005 to $15.7 \%$ in 2014 [3]. The customary Korean diet is characterized by a high proportion of carbohydrate consumption compared to the average Western diet. Based on results from the 2014 Korea National Health and Nutrition Examination Survey, the proportion of carbohydrate consumption was $63.0 \%$ for males and $65.8 \%$ for females [4]. Carbohydrate is consumed not only through regular meals but also from snacks. The most popular carbohydrate originated snacks among Koreans include cereals, ramen, rice cakes, and bread.

Effects of walnut (Juglans) intake on various heart health markers, including reducing total cholesterol (TC), lowering low-density lipoprotein cholesterol (LDL-C) [5], decreasing blood pressure [6], and improving vascular function [7], have been investigated. A meta-analysis [8] reviewed the heart health benefits of walnuts on 365 participants, and compared to control diets, diets that were supplemented with walnuts $(5 \%-10 \%$ of total calories) resulted in a significantly greater

This work was supported by California Walnut Commission (2-1510-0001).

${ }^{\S}$ Corresponding Author: Hyunjin Park, Tel. 82-2-780-1208, Fax. 82-2-785-1208, Email. gracepark06@gmail.com

Received: July 16, 2018, Revised: September 15, 2018, Accepted: December 17, 2018

This is an Open Access article distributed under the terms of the Creative Commons Attribution Non-Commercial License (http://creativecommons.org/licenses/by-nc/3.0/) which permits unrestricted non-commercial use, distribution, and reproduction in any medium, provided the original work is properly cited. 
decrease in TC and LDL-C without any adverse effect on body weight. Compared to the favorable results for walnut intake on heart health, there are limited data for the effects of walnut consumption on MetS. A double-blinded, randomized, placebocontrolled study [9] found that consuming $48 \mathrm{~g}$ of walnuts each day for four days may help to control blood lipids among subjects with MetS. Additional research [10] reported that daily ingestion of $56 \mathrm{~g}$ of walnuts improves endothelial function in overweight adults with visceral adiposity, which is one of the components of MetS. Results showing the effects of nut consumption, including walnuts, on improving MetS components have been inconsistent. There were no significant improvement of MetS indicators among South African subjects after a walnut feeding study provided $20 \%$ of calories from walnut intake for 8 weeks [11], whereas improvement of insulin resistance has been reported among Spanish subjects with MetS after 12 weeks of daily mixed nut consumption, which included $15 \mathrm{~g}$ of walnuts [12].

With the need for longer-term studies in the area of MetS and to elucidate the effects of walnut consumption, we conducted a two-arm, randomized, controlled crossover trial to evaluate the effects of walnut consumption on the management of MetS components. In addition, we investigated lipid profiles and circulating markers of insulin resistance, adiponectin, and leptin in subjects with MetS.

\section{SUBJECTS AND METHODS}

\section{Subjects}

Participants were recruited through an online community platform and through local newspaper advertisements in Seoul, South Korea. Among 210 screened volunteers, 119 (aged 30-55 years) eligible volunteers were enrolled in the study. According to the revised NCEP criteria [2], each participant was diagnosed as having MetS if he or she had three or more of the following criteria: 1) waist circumference $\geq 90 \mathrm{~cm}$ for men and $\geq 80 \mathrm{~cm}$ for women; 2) triglycerides $\geq 150 \mathrm{mg} / \mathrm{dL}$; 3) $\mathrm{HDL}$ cholesterol $<40 \mathrm{mg} / \mathrm{dL}$ for men and < $50 \mathrm{mg} / \mathrm{dL}$ for women; 4) blood pressure $\geq 130 / 85 \mathrm{mmHg}$; and 5) fasting glucose $\geq 100 \mathrm{mg} / \mathrm{dL}$. Exclusion criteria were: body mass index (BMI) greater than 35; the use of any medications to control blood pressure, lipid metabolism, or glucose; consumption of a hypocaloric diet within the past year and regular consumption of omega-3 supplements; and post-menopausal women and smokers. All study procedures were approved by the Ethics Committee of Sookmyung Women's University, Seoul, South Korea (SMWU1508-BR-051-01). All of the participants provided written informed consent before the start of the interventions, and the study was conducted according to the principles of the Declaration of Helsinki.

Assessed for eligibility $(n=271)$

At least 3 risk factors out of 5 metabolic syndrome indices

- Waist circumference $\geq 90 \mathrm{~cm}$ in men, $\geq 85 \mathrm{~cm}$ in women

Blood pressure $\geq 130 / 85 \mathrm{mmHg}$

- Blood triglyceride level $\geq 150 \mathrm{mg} / \mathrm{dL}$

Blood HDL-cholesterol level $\leq 40 \mathrm{mg} / \mathrm{dL}$ in men, $\leq 50 \mathrm{mg} / \mathrm{dL}$ in women

- Fasting blood glucose level $\geq 100 \mathrm{mg} / \mathrm{dL}$

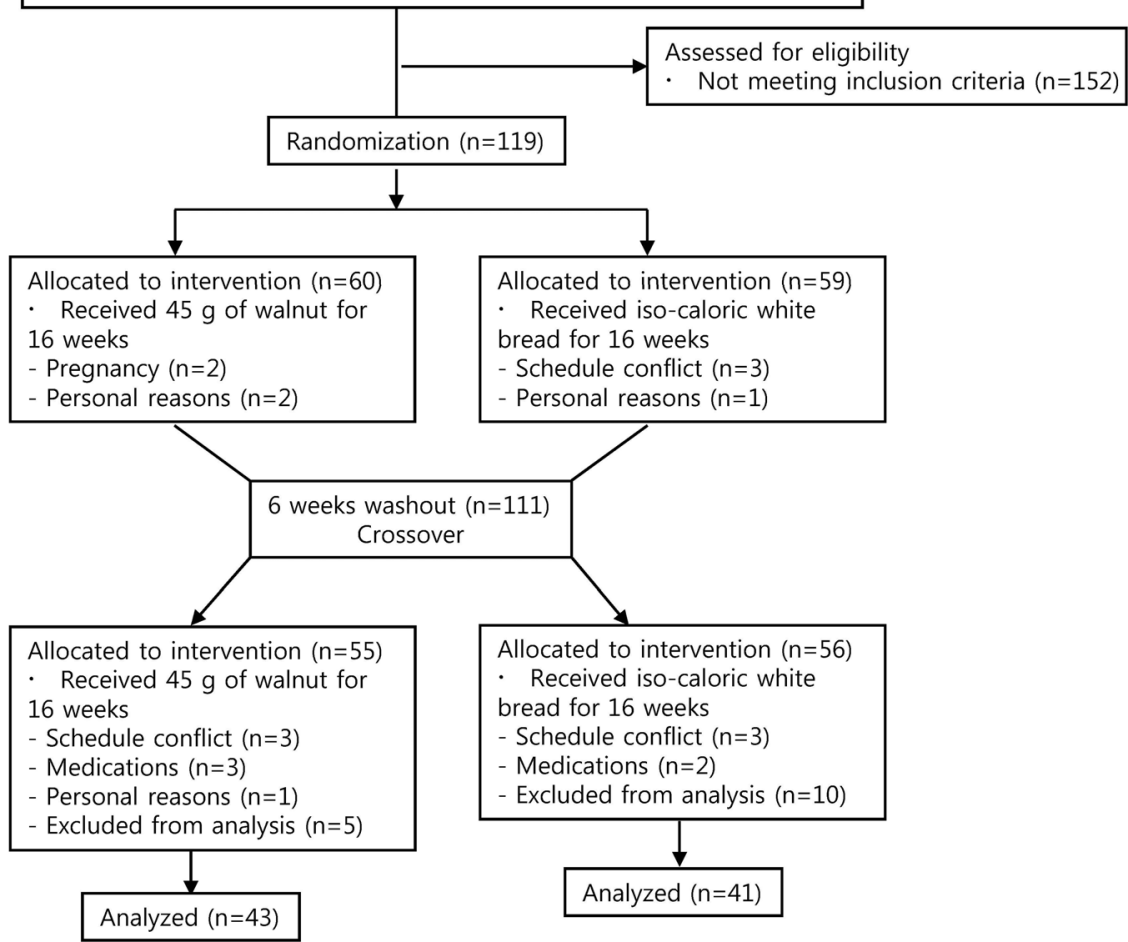

Fig. 1. CONSORT flow chart diagram of the crossover trial 


\section{Study design}

A randomized, controlled, crossover, 16 week intervention was conducted using $45 \mathrm{~g}$ of walnut and iso-caloric white bread treatments. A 6 week washout period was included between the 16 week consumptions of each treatment. The subjects were randomly assigned to one of two groups by using a random-number generator program. Subjects in the first group ( $n=60$ ) were instructed to consume $45 \mathrm{~g}$ of walnut (containing $305.4 \mathrm{kcal}, 6.4 \mathrm{~g}$ of carbohydrate, $28.9 \mathrm{~g}$ of fat, and $6.4 \mathrm{~g}$ of protein) per day for 16 weeks, as convenient during the daytime. After a 6 week washout period, those subjects consumed white bread (corresponding to $313.3 \mathrm{kcal}, 60.5 \mathrm{~g}$ of carbohydrate, $3.4 \mathrm{~g}$ of fat, and $10.2 \mathrm{~g}$ of protein) per day for 16 weeks, as convenient during the daytime. The second group $(n=59)$ received white bread for 16 weeks followed by a 6 week washout period, after which they received walnuts for 16 weeks. Fig. 1 shows the process from screening to crossover randomization of the participants. Thirty subjects were unable to finish the study due to the length of the intervention period, pregnancy, or use of medication. Thus, a total of 84 volunteers completed the intervention.

\section{Diet and physical activity assessment}

Three-day dietary records that included two consecutive weekdays and one weekend day were completed once before each intervention and twice during the trial period. A registered dietitian provided detailed instructions to participants on how to fill out the diet record and report their diet by taking a picture and sending it via text messages. The dietary intake of the participants was assessed based on the three-day dietary record before the trial and one record during each period. The data were processed using CAN-Pro 4.0 software (The Korea Nutrition Society). Each participant was provided a consumption log sheet to self-report compliance with protocols. Subjects recorded how many packages they did not consume during the previous week and reported the number to care providers each Monday using a text message. Physical activity was estimated by using the international physical activity questionnaire short form. Maintaining a habitual diet and physical activity level were required to prevent intentional weight gain or loss during the study period.

\section{Measurements}

During the study, participants visited the clinical research unit four times: once at baseline, once after the washout period, and at the end of both intervention periods. Anthropometric measurements and blood sample collection were conducted during each visit.

\section{Anthropometric and body composition measurement}

Each of the anthropometric measurements was obtained by a single trained examiner while the participant was barefoot. Waist and hip circumference measurements were obtained by using a measuring tape (SECA-201; SECA, Hamburg, Germany). Body weight was measured and rounded to the nearest 0.1 $\mathrm{kg}$, and height was obtained by using a stadiometer (BSM330; Biospace, Seoul, Korea) and rounded to the nearest $0.1 \mathrm{~cm}$. Blood pressure was measured on the right arm using an up-load blood pressure monitor (BPBIO320S; Biospace, Seoul, Korea) after at least a 5 min rest period. Body composition including body composition analysis (total body water, protein, mineral, and body fat mass), muscle-fat analysis (body weight, skeletal muscle mass, and body fat mass), obesity analysis [percentage body fat and BMI (calculated as weight $(\mathrm{kg})$ divided by height in meters ( $m$ ) squared)] was assessed by undertaking multifrequency whole-body bioimpedance measurements using InBody 620 (Biospace, Seoul, Korea).

\section{Blood sampling and determination of general serum biochemical variables}

Blood samples were collected in the fasting state and the samples were immediately centrifuged at 3,000 r/min for 15 min at $4^{\circ} \mathrm{C}$. Serum and plasma specimens were frozen at $-80^{\circ} \mathrm{C}$ until analysis. Serum TC and TG levels were measured by using an enzymatic-colorimetric method, HDL-C and LDL-C levels were determined via a homogeneous enzymatic-colorimetry method, and the high-sensitive C-reactive protein (hs-CRP) level was measured by applying a particle-enhanced immunoturbidimetric method using a Cobas 8000 c702 chemistry analyzer (Roche Diagnostics, Mannheim, Germany). Fasting blood glucose was measured using a Glucocard X-meter (Arkray, Kyoto, Japan). Serum insulin was measured with the commercially available, ultrasensitive insulin ELISA kit (80-INSHU-E01.1, E10.1; ALPCO, Salem, NH, USA) using an Epoch microplate spectrophotometer (BIOTEK, Winooski, VT, USA).

Serum leptin and adiponectin levels were measured using the quantikine human leptin ELISA kit (DLP00; R\&D Systems, Minneapolis, MN, USA) and the quantikine human total adiponectin ELISA kit (DRP300; R\&D Systems, Minneapolis, MN, USA), respectively. Hemoglobin A1c (HbA1c) separation and quantification were performed by using a high-performance liquid chromatography analyzer (Tosoh HLC-723 G8; Sysmex, Kobe, Japan). Serum apolipoprotein B (apoB) was measured by turbidimetric immunoassay using a HITACHI 7600 chemistry analyzer (Hitachi, Tokyo, Japan).

\section{Plasma fatty acid composition}

Total lipid extraction from plasma was performed by applying a modified Folch method [13], and fatty acid methyl esters (FAMEs) were obtained via the modified method described by William et al. [14]. FAME samples were analyzed by an Agilent 7890B gas chromatograph (Agilent Technologies, Folsom, CA, USA) equipped with a flame ionization detector and a DB-WAX capillary column (J \& W Scientific, Folsom, CA, USA) (30 m long, $0.25 \mu \mathrm{m}$ film thickness, and $0.25 \mathrm{~mm}$ inner diameter). For analysis, a $2 \mu \mathrm{L}$ sample was manually injected into the inlet at $250^{\circ} \mathrm{C}$ in a $20: 1$ pulsed split mode under 15 psi. Initial oven temperature was $150^{\circ} \mathrm{C}$ for $1 \mathrm{~min}$ followed by heating to $180^{\circ} \mathrm{C}$ at a rate of $10^{\circ} \mathrm{C} / \mathrm{min}$, then to $235^{\circ} \mathrm{C}$ at a rate of $2^{\circ} \mathrm{C} / \mathrm{min}$, finally to $245^{\circ} \mathrm{C}$ at a rate of $1^{\circ} \mathrm{C} / \mathrm{min}$, and then maintained for $1 \mathrm{~min}$. Carrier gas $\left(\mathrm{N}_{2}\right)$, air, fuel gas $\left(\mathrm{H}_{2}\right)$, and make-up gas $\left(\mathrm{N}_{2}\right)$ flowed at speeds of $1 \mathrm{~mL} / \mathrm{min}, 350 \mathrm{~mL} / \mathrm{min}, 30 \mathrm{~mL} / \mathrm{min}$, and $20 \mathrm{~mL} / \mathrm{min}$, respectively. The detector was set at $270^{\circ} \mathrm{C}$. Each FAME was identified by comparison with a multiple FAME mix standard and several single standards. FAMEs were quantitated by applying a single point internal standard method using Agilent ChemStation software. 


\section{Statistical analysis}

We estimated the appropriate sample number by taking into consideration the specific variances of methodology for MetS components with a type I error of $a=0.05$ and $80 \%$ power. After verifying the efficacy of the washout period by comparing the initial baseline and post-washout baseline data, we merged the results from both arms of the interventions ( $n=84$ each). To identify the significant differences between groups that started with either walnut $(n=43)$ or control (bread; $n=41)$ at baseline, an independent samples $t$-test was conducted. After verifying the efficacy of the washout period using a paired $t$-test, the initial baseline and post-washout baseline data were combined and the final data from each study arm ( $n=84$ each for the two interventions) were consolidated. Treatment effects for each intervention were determined using a paired $t$-test on each variable. Intervention effects were determined using a linear mixed-effects model with the intercept as a random effect and a covariance structure for repeated measures by time and treatment. The fixed effects included time as a categorical variable, interventions, age, sex, BMI, WC, energy intake, and interactions between intervention and the arms. After 16 weeks of walnut consumption, we calculated the proportion of participants who no longer met the criteria for MetS (i.e., reverted MetS). Values are presented as mean \pm SD and a $P<$ 0.05 was considered significant. All statistical analyses were performed using SPSS (IBM SPSS version 23, IBM corp., Armonk, NY, USA).

\section{RESULTS}

Preintervention characteristics and fatty acid profiles of subjects by group

Before the intervention, the average age of the participants who consumed walnut first (WAL) was greater than that of the subjects who consumed control food (bread) first (CON). In addition, the average body fat mass of WAL at baseline was significantly greater than that of CON. Consequently, TG, LDL-C, insulin, apoB, and hs-CRP levels were significantly higher in the WAL group, whereas the HDL-C level was significantly lower in the WAL group, as shown in Table 1.

Table 1. Baseline characteristics of metabolic syndrome components, body composition, and blood metabolic profile among the subjects with metabolic syndrome.

\begin{tabular}{|c|c|c|c|}
\hline & ALL $(n=84)$ & $\operatorname{CON}(n=41)$ & WAL $(n=43)$ \\
\hline Age (yrs) & $39.44 \pm 6.53$ & $37.91 \pm 5.69$ & $41.05 \pm 7.02^{*}$ \\
\hline Men, n (\%) & $42(50)$ & $20(48.8)$ & $22(51.2)$ \\
\hline BMI $\left(k g / m^{2}\right)$ & $27.08 \pm 3.61$ & $26.29 \pm 3.31$ & $27.92 \pm 3.75$ \\
\hline \multicolumn{4}{|l|}{ MetS components } \\
\hline Systolic blood pressure (mm Hg) & $134.51 \pm 14.47$ & $134.21 \pm 16.38$ & $134.83 \pm 12.35$ \\
\hline Diastolic blood pressure $(\mathrm{mm} \mathrm{Hg})$ & $83.32 \pm 10.74$ & $83.51 \pm 10.90$ & $83.12 \pm 10.71$ \\
\hline Waist circumference $(\mathrm{cm})$ & $89.87 \pm 7.75$ & $90.06 \pm 6.31$ & $89.67 \pm 9.15$ \\
\hline Triglycerides (mg/dL) & $135.9 \pm 65.09$ & $111.60 \pm 53.71$ & $162.08 \pm 66.71^{*}$ \\
\hline HDL cholesterol (mg/dL) & $41.58 \pm 9.01$ & $44.12 \pm 10.39$ & $38.98 \pm 6.49^{*}$ \\
\hline Fasting glucose (mg/dL) & $104.94 \pm 25.45$ & $104.40 \pm 7.63$ & $105.51 \pm 35.80$ \\
\hline \multicolumn{4}{|l|}{ Body composition } \\
\hline Body weight (kg) & $76.09 \pm 14.85$ & $73.70 \pm 12.20$ & $78.61 \pm 16.99$ \\
\hline Waist-hip ratio & $0.92 \pm 0.06$ & $0.93 \pm 0.05$ & $0.92 \pm 0.06$ \\
\hline Total body water (L) & $37.65 \pm 7.82$ & $37.25 \pm 7.11$ & $38.08 \pm 8.57$ \\
\hline Total body protein $(\mathrm{kg})$ & $10.15 \pm 2.13$ & $10.07 \pm 1.95$ & $10.24 \pm 2.34$ \\
\hline Body mineral (kg) & $3.53 \pm 0.75$ & $3.44 \pm 0.69$ & $3.62 \pm 0.81$ \\
\hline Body fat mass (kg) & $24.75 \pm 7.66$ & $22.93 \pm 6.78$ & $26.66 \pm 8.14^{*}$ \\
\hline Soft lean mass (kg) & $48.42 \pm 10.09$ & $47.91 \pm 9.17$ & $48.96 \pm 11.06$ \\
\hline Fat free mass $(\mathrm{kg})$ & $51.34 \pm 10.69$ & $50.77 \pm 9.72$ & $51.96 \pm 11.70$ \\
\hline Skeletal muscle mass (kg) & $28.65 \pm 6.47$ & $28.36 \pm 5.90$ & $28.95 \pm 7.08$ \\
\hline Percent body fat (\%) & $32.4 \pm 6.85$ & $31.09 \pm 6.93$ & $33.80 \pm 6.57$ \\
\hline \multicolumn{4}{|l|}{ Metabolic profile } \\
\hline LDL cholesterol (mg/dL) & $116.27 \pm 28.92$ & $107.67 \pm 25.93$ & $125.29 \pm 29.45^{*}$ \\
\hline Total cholesterol (mg/dL) & $191.19 \pm 34.67$ & $195.74 \pm 36.05$ & $186.54 \pm 32.98$ \\
\hline Insulin (pmol/L) & $2.71 \pm 1.51$ & $2.06 \pm 1.32$ & $3.35 \pm 1.43^{*}$ \\
\hline HbA1c (\%) & $5.38 \pm 0.345$ & $5.34 \pm 0.40$ & $5.42 \pm 0.28$ \\
\hline ApoB (mg/dL) & $96.04 \pm 19.09$ & $91.33 \pm 18.07$ & $100.75 \pm 19.14^{*}$ \\
\hline hs-CRP (mg/L) & $0.83 \pm 0.68$ & $0.63 \pm 0.45$ & $1.05 \pm 0.81^{*}$ \\
\hline Adiponectin $(\mu \mathrm{g} / \mathrm{mL})$ & $4.31 \pm 2.08$ & $4.27 \pm 1.99$ & $4.35 \pm 2.19$ \\
\hline Leptin $(\mu \mathrm{g} / \mathrm{mL})$ & $15.30 \pm 6.72$ & $13.78 \pm 5.67$ & $16.78 \pm 7.37^{*}$ \\
\hline
\end{tabular}

CON, subjects who consumed white bread first; WAL, subjects who consumed walnut first; BMl, body mass index; MetS, metabolic syndrome; HbA1c, hemoglobin A1c; $\mathrm{ApoB}$, apolipoprotein $\mathrm{B}$; hs-CRP, high sensitivity C-reactive protein.

Data are mean $\pm \mathrm{SD}$

* indicates a significant difference between CON and WAL groups using independent $t$-test 
Table 2. Baseline blood fatty acid profiles (\%) in subjects with metabolic syndrome.

\begin{tabular}{|c|c|c|c|}
\hline & ALL $(n=84)$ & $\operatorname{CON}(n=41)$ & WAL $(n=43)$ \\
\hline Myristic acid & $1.72 \pm 0.76$ & $1.81 \pm 0.71$ & $1.64 \pm 0.81$ \\
\hline Palmitic acid & $56.85 \pm 3.85$ & $56.81 \pm 4.17$ & $56.90 \pm 3.57$ \\
\hline Stearic acid & $21.73 \pm 3.15$ & $22.99 \pm 3.39$ & $20.54 \pm 2.38^{*}$ \\
\hline Oleic acid & $6.58 \pm 1.99$ & $6.34 \pm 2.20$ & $6.80 \pm 1.77$ \\
\hline Linoleic acid & $7.54 \pm 2.52$ & $6.72 \pm 2.21$ & $8.32 \pm 2.58^{*}$ \\
\hline Gamma-linolenic acid & $0.8 \pm 0.32$ & $0.92 \pm 0.36$ & $0.69 \pm 0.22^{*}$ \\
\hline Alpha-linolenic acid & $0.25 \pm 0.35$ & $0.34 \pm 0.40$ & $0.17 \pm 0.27^{*}$ \\
\hline Arachidonic acid & $2.43 \pm 1.01$ & $2.16 \pm 0.98$ & $2.69 \pm 0.98^{*}$ \\
\hline Eicosapentaenoic acid & $0.55 \pm 0.65$ & $0.47 \pm 0.76$ & $0.62 \pm 0.54$ \\
\hline Docosahexaenoic acid & $1.51 \pm 0.96$ & $1.42 \pm 0.97$ & $1.60 \pm 0.94$ \\
\hline Total SFAs & $80.31 \pm 5.03$ & $81.61 \pm 4.62$ & $79.08 \pm 5.14^{*}$ \\
\hline Total MUFAs & $6.59 \pm 1.98$ & $6.34 \pm 2.20$ & $6.83 \pm 1.73$ \\
\hline Total PUFAs & $13.19 \pm 4.31$ & $12.05 \pm 3.85$ & $14.26 \pm 4.48^{*}$ \\
\hline Total $n-3$ PUFAs & $2.34 \pm 1.54$ & $2.26 \pm 1.46$ & $2.43 \pm 1.64$ \\
\hline Total $n-6$ PUFAs & $10.77 \pm 3.25$ & $9.79 \pm 2.88$ & $11.69 \pm 3.35^{*}$ \\
\hline
\end{tabular}

CON, subjects who consumed white bread first; WAL, subjects who consumed walnut first; SFAs, saturated fatty acids; MUFAs, monounsaturated fatty acids; PUFAs, polyunsaturated fatty acids.

Fatty acid compositions are shown as a percentage of the total amount of fatty acids measured.

Data are mean $\pm \mathrm{SD}$

* indicates a significant difference between CON and WAL groups using independent $t$-test.

Table 3. Dietary intake of the participants at baseline and after the 16 week walnut intake period

\begin{tabular}{|c|c|c|c|c|}
\hline & Before walnut intervention (1) & During walnut intervention (2) & (2)-(1) & $P$ \\
\hline Energy (kcal) & $1,683.22 \pm 334.37$ & $1,652.94 \pm 409.79$ & $-30.28 \pm 51.58$ & 0.559 \\
\hline Carbohydrate (g) & $233.9 \pm 48.74$ & $217.35 \pm 56.14$ & $-16.55 \pm 6.35$ & 0.011 \\
\hline Total fat (g) & $48.89 \pm 15.93$ & $55.25 \pm 19.08$ & $6.37 \pm 2.55$ & 0.015 \\
\hline Vegetable fat (g) & $24.16 \pm 9.08$ & $33.04 \pm 14.90$ & $8.88 \pm 1.74$ & $<0.0001$ \\
\hline Animal fat (g) & $24.73 \pm 11.14$ & $22.22 \pm 10.66$ & $-2.51 \pm 1.61$ & 0.122 \\
\hline Total protein (g) & $69.58 \pm 18.6$ & $66.08 \pm 17.34$ & $-3.50 \pm 2.50$ & 0.164 \\
\hline Vegetable protein (g) & $28.92 \pm 7.93$ & $29.42 \pm 8.22$ & $0.50 \pm 1.16$ & 0.665 \\
\hline Animal protein $(\mathrm{g})$ & $40.66 \pm 15.76$ & $36.66 \pm 13.97$ & $-4.00 \pm 2.06$ & 0.056 \\
\hline Fiber (g) & $17.95 \pm 5.75$ & $17.21 \pm 5.16$ & $-0.74 \pm 0.64$ & 0.250 \\
\hline Cholesterol (mg) & $370.11 \pm 155.31$ & $345.97 \pm 137.67$ & $-24.13 \pm 21.93$ & 0.274 \\
\hline Total fatty acid (g) & $28.17 \pm 14.4$ & $35.11 \pm 16.54$ & $6.94 \pm 2.34$ & 0.004 \\
\hline SFAs (g) & $8.97 \pm 5.96$ & $9.32 \pm 5.41$ & $0.35 \pm 0.87$ & 0.693 \\
\hline MUFAs (g) & $11.18 \pm 7.11$ & $11.6 \pm 5.72$ & $0.42 \pm 0.98$ & 0.669 \\
\hline PUFAs (g) & $9.31 \pm 4.48$ & $15.21 \pm 9.42$ & $5.90 \pm 1.09$ & $<0.0001$ \\
\hline Omega-3 fatty acid (g) & $0.84 \pm 0.46$ & $1.66 \pm 1.22$ & $0.82 \pm 0.14$ & $<0.0001$ \\
\hline
\end{tabular}

SFAs, saturated fatty acids; MUFAs, monounsaturated fatty acids; PUFAs, polyunsaturated fatty acids.

Data are mean $\pm \mathrm{SD}$.

Statistical significance set at $P<0.05$ for the difference between dietary intake before consuming walnut and during walnut intake using a two-tailed paired $t$-test.

$P$-values for comparison between pre- and post-intervention using paired $t$-tests.

Blood fatty acid profiles at baseline are presented in Table 2. Subjects in the WAL group had significantly higher sums of polyunsaturated fatty acids (PUFAs) and $n-6$ fatty acids but a significantly lower sum of saturated fatty acids (SFAs) compared to those of the CON group.

\section{Dietary analysis}

Dietary analysis results before and during walnut intervention are presented in Table 3. Subjects maintained an iso-caloric diet during the walnut intake period. Compared with the pre-walnut intake, consumption of carbohydrate was significantly reduced and total fat intake, particularly vegetable fat intake, was increased during the intervention period. Intake of total fatty acids during the intervention period was increased due to the significant increase in PUFAs intake. No changes in dietary intake, including calorie intake, were observed between preand post-control food intake (data not shown).

Effects of walnut intake on MetS components and its prevalence

Reversion rates for MetS after daily walnut intake for 16 weeks are presented in Table 4. At baseline, all 84 subjects exhibited MetS. However, after 16 weeks of walnut intervention, 43 subjects had reverted to normal status. The number of subjects whose MetS risk factors were reduced after walnut intake was 
Table 4. Prevalence of metabolic syndrome and its components among study participants at baseline and after the 16 week walnut intake period ${ }^{1)}$

\begin{tabular}{|c|c|}
\hline \multicolumn{2}{|l|}{ MetS } \\
\hline Baseline & $84(100.0)$ \\
\hline PostW ${ }^{2)}$ Reduced number of risk factors & $54(64.3)$ \\
\hline Reversion rate $\mathrm{r}^{3)}$ & $43(51.2)$ \\
\hline \multicolumn{2}{|l|}{ Central obesity } \\
\hline Baseline & $69(82.2)$ \\
\hline PostW & $37(44.0)$ \\
\hline Reversion rate & $32(46.4)$ \\
\hline \multicolumn{2}{|l|}{ Elevated fasting glucose } \\
\hline Baseline & $56(66.7)$ \\
\hline PostW & $47(56.0)$ \\
\hline Reversion rate & 19 (33.9) \\
\hline \multicolumn{2}{|l|}{ Elevated triglycerides } \\
\hline Baseline & $36(42.9)$ \\
\hline PostW & $23(27.4)$ \\
\hline Reversion rate & $19(52.8)$ \\
\hline \multicolumn{2}{|l|}{ Reduced HDL cholesterol } \\
\hline Baseline & $65(77.4)$ \\
\hline PostW & $46(54.8)$ \\
\hline Reversion rate & $22(33.8)$ \\
\hline \multicolumn{2}{|l|}{ Elevated systolic blood pressure } \\
\hline Baseline & $56(66.7)$ \\
\hline PostW & $40(47.6)$ \\
\hline Reversion rate & $16(28.6)$ \\
\hline \multicolumn{2}{|l|}{ Elevated diastolic blood pressure } \\
\hline Baseline & $46(54.8)$ \\
\hline PostW & $26(31.0)$ \\
\hline Reversion rate & 15 (32.6) \\
\hline \multicolumn{2}{|c|}{$\begin{array}{l}\text { MetS: metabolic syndrome } \\
\text { 1) Data are } \mathrm{n}(\%) \text {. } \\
\text { 2) PostW, the period after consuming walnut for } 16 \text { weeks. } \\
\text { 3) Reversion rate indicates the proportion of participants who met the criterion at } \\
\text { baseline but not after walnut consumption. }\end{array}$} \\
\hline
\end{tabular}

even greater than the number of reverted subjects. Of the participants, $64.3 \%$ experienced improvement in one or more MetS components. Central obesity and reduced HDL-C were the most common components among study participants at baseline (82.2\% and $77.4 \%$, respectively) and their reversion rates after walnut intake were $46.4 \%$ and $33.8 \%$, respectively. Over $60 \%$ of the study participants showed elevated fasting glucose level and blood pressure at baseline. However, about 30\% of the subjects had reverted back to normal glucose level and blood pressure status after the walnut intervention. Subjects with an elevated TG level comprised $42.9 \%$ of the volunteers at baseline, which was the lowest prevalence among the five MetS components assessed. However, the TG reversion rate was the highest (52.8\%).

Baseline characteristics before each intervention are summarized in Table 5; none of the parameters differed between the two baseline periods. There were no significant differences between groups in reported physical activity (data not shown). However, we detected significant intervention effects on HDL-C ( $P=$ $0.028)$ and fasting glucose $(P=0.000)$, compared to the control group after applying linear mixed model adjustments for age, gender, BMI, and sequence (Table 5). The HDL-C level was increased after walnut intervention but was stable after control intervention. A significant intervention effect on fasting blood glucose was observed after walnut intake compared with that after the control intervention, but this might be due to an increase in glucose level after the control period. Other MetS components including blood pressure, WC, and TG were reduced after walnut intake, although there were no significant intervention effects compared to those after the control intervention.

Table 5. Metabolic syndrome components and blood metabolic profiles in subjects with metabolic syndrome at baseline and after the 16 week walnut and white bread (control) interventions

\begin{tabular}{|c|c|c|c|c|c|}
\hline & $\begin{array}{l}\text { Before white bread } \\
\text { intervention }\end{array}$ & $\begin{array}{l}\text { After white bread } \\
\text { intervention }\end{array}$ & $\begin{array}{l}\text { Before walnut } \\
\text { intervention }\end{array}$ & $\begin{array}{l}\text { After walnut } \\
\text { intervention }\end{array}$ & $P$ \\
\hline \multicolumn{6}{|l|}{ MetS components } \\
\hline Systolic blood pressure (mm Hg) & $133.93 \pm 15.47$ & $129.16 \pm 14.72^{*}$ & $134.92 \pm 15.11$ & $131.85 \pm 14.82^{*}$ & 0.054 \\
\hline Diastolic blood pressure (mm Hg) & $83.46 \pm 10.40$ & $79.49 \pm 11.36$ & $81.32 \pm 11.44$ & $81.25 \pm 11.47$ & 0.813 \\
\hline Waist circumference $(\mathrm{cm})$ & $89.01 \pm 7.70$ & $85.76 \pm 9.58^{*}$ & $88.42 \pm 8.90$ & $86.83 \pm 9.11^{*}$ & 0.693 \\
\hline Triglycerides (mg/dL) & $124.11 \pm 56.47$ & $135.21 \pm 58.23$ & $137.93 \pm 64.83$ & $117.74 \pm 51.78^{*}$ & 0.219 \\
\hline HDL cholesterol (mg/dL) & $41.61 \pm 9.37$ & $41.76 \pm 9.55$ & $42.36 \pm 9.32$ & $43.86 \pm 10.03$ & 0.028 \\
\hline Fasting glucose (mg/dL) & $106.27 \pm 24.62$ & $111.58 \pm 30.20$ & $103.04 \pm 27.44$ & $101.37 \pm 16.46$ & 0.000 \\
\hline \multicolumn{6}{|l|}{ Metabolic profile } \\
\hline LDL cholesterol (mg/dL) & $116.38 \pm 28.51$ & $118.76 \pm 29.71$ & $121.74 \pm 28.09$ & $113.07 \pm 23.63$ & 0.866 \\
\hline Total cholesterol (mg/dL) & $191.78 \pm 34.61$ & $187.15 \pm 32.01$ & $185.17 \pm 30.63$ & $178.28 \pm 30.34^{*}$ & 0.633 \\
\hline Insulin (pmol/L) & $2.67 \pm 1.55$ & $2.55 \pm 1.17$ & $2.77 \pm 1.43$ & $2.61 \pm 1.46$ & 0.510 \\
\hline HbA1c (\%) & $5.38 \pm 0.37$ & $5.43 \pm 0.37^{*}$ & $5.39 \pm 0.34$ & $5.38 \pm 0.35$ & 0.021 \\
\hline ApoB (mg/dL) & $93.57 \pm 18.27$ & $89.76 \pm 20.49$ & $94.23 \pm 19.32$ & $89.45 \pm 19.17^{*}$ & 0.108 \\
\hline hs-CRP (mg/L) & $0.81 \pm 0.64$ & $0.78 \pm 0.66$ & $0.90 \pm 0.74$ & $0.83 \pm 0.66$ & 0.185 \\
\hline Adiponectin $(\mu \mathrm{g} / \mathrm{mL})$ & $4.10 \pm 2.12$ & $4.02 \pm 1.88$ & $4.51 \pm 2.10$ & $4.99 \pm 1.91$ & 0.019 \\
\hline Leptin $(\mu \mathrm{g} / \mathrm{mL})$ & $16.18 \pm 7.79$ & $12.38 \pm 8.17$ & $14.65 \pm 6.87$ & $15.64 \pm 6.96$ & 0.853 \\
\hline
\end{tabular}

MetS, metabolic syndrome; HbA1c, hemoglobin A1c; ApoB, apolipoprotein B; hs-CRP, high sensitivity C-reactive protein.

Data are mean $\pm \mathrm{SD}$

$P$-values of significance between walnut and control determined using a linear mixed model and after adjustment for age, gender, BMl, and sequence.

* indicates significant difference $(P<0,05)$ between pre- and post- of each intervention type using a paired $t$-test. 
Table 6. Blood fatty acid profiles (\%) in subjects with metabolic syndrome at baseline and after the 16 week walnut and white bread interventions

\begin{tabular}{|c|c|c|c|c|c|}
\hline & $\begin{array}{l}\text { Before white bread } \\
\text { intervention }\end{array}$ & $\begin{array}{l}\text { After white bread } \\
\text { intervention }\end{array}$ & $\begin{array}{l}\text { Before walnut } \\
\text { intervention }\end{array}$ & $\begin{array}{l}\text { After walnut } \\
\text { intervention }\end{array}$ & $P$ \\
\hline Myristic acid & $2.51 \pm 1.74$ & $2.40 \pm 1.69$ & $2.23 \pm 1.98$ & $2.00 \pm 1.50$ & 0.000 \\
\hline Palmitic acid & $58.22 \pm 3.90$ & $54.23 \pm 4.67^{*}$ & $56.70 \pm 4.44$ & $56.35 \pm 3.10$ & 0.103 \\
\hline Stearic acid & $23.13 \pm 2.93$ & $24.87 \pm 2.67$ & $22.92 \pm 3.69$ & $24.25 \pm 2.54^{*}$ & 0.088 \\
\hline Oleic acid & $6.13 \pm 2.44$ & $6.33 \pm 2.09$ & $6.25 \pm 1.94$ & $6.61 \pm 2.35$ & 0.375 \\
\hline Linoleic acid & $5.48 \pm 2.13$ & $5.98 \pm 2.59$ & $6.09 \pm 3.04$ & $5.64 \pm 2.46$ & 0.340 \\
\hline Gamma-linolenic acid & $0.87 \pm 0.28$ & $0.58 \pm 0.32^{*}$ & $0.80 \pm 0.22$ & $0.67 \pm 0.27^{*}$ & 0.431 \\
\hline Alpha-linolenic acid & $0.21 \pm 0.33$ & $0.22 \pm 0.25$ & $0.23 \pm 0.30$ & $0.29 \pm 0.28^{*}$ & 0.012 \\
\hline Arachidonic acid & $1.66 \pm 0.89$ & $2.22 \pm 1.49$ & $1.90 \pm 1.12$ & $1.61 \pm 1.05$ & 0.743 \\
\hline Eicosapentaenoic acid & $0.80 \pm 1.01$ & $1.56 \pm 1.08$ & $1.63 \pm 1.17$ & $1.40 \pm 0.96$ & 0.967 \\
\hline Docosahexaenoic acid & $0.99 \pm 0.88$ & $1.52 \pm 1.36$ & $1.23 \pm 0.87$ & $1.17 \pm 0.72$ & 0.716 \\
\hline Total SFAs & $83.86 \pm 4.82$ & $81.50 \pm 5.00$ & $81.85 \pm 5.22$ & $82.60 \pm 4.05$ & 0.431 \\
\hline Total MUFAs & $6.13 \pm 2.44$ & $6.33 \pm 2.09$ & $6.27 \pm 1.93$ & $6.61 \pm 2.35^{*}$ & 0.354 \\
\hline Total PUFAs & $10.01 \pm 3.63$ & $12.17 \pm 4.28$ & $11.97 \pm 4.24$ & $10.79 \pm 3.62$ & 0.485 \\
\hline Total n-3 PUFAs & $2.01 \pm 1.42$ & $3.38 \pm 1.64$ & $3.11 \pm 1.55$ & $2.86 \pm 1.27$ & 0.459 \\
\hline Total n-6 PUFAs & $8.00 \pm 2.83$ & $8.79 \pm 3.81$ & $8.79 \pm 3.97$ & $7.93 \pm 3.30$ & 0.582 \\
\hline
\end{tabular}

SFAs, saturated fatty acids; MUFAs, monounsaturated fatty acids; PUFAs, polyunsaturated fatty acids.

Fatty acid compositions are shown as a percentage of the total amount of fatty acids measured.

Data are mean $\pm \mathrm{SD}$

$P$-values of significance between walnut and control determined using a linear mixed model and adjusting for age, gender, BMl, and sequence.

\section{Effects of walnut intake on metabolic profiles}

Baseline and final levels of the MetS components and metabolic profiles are presented in Table 5. None of the parameters were significantly different between the pre-walnut and pre-control periods. When analyzed using a paired $t$-test, it was noticed that TC and apoB had decreased after walnut consumption for 16 weeks. Significant differences in treatment effect between the two interventions on $\mathrm{HbA1c}(P=0.021)$, and adiponectin $(P=0.019)$ were observed after linear mixed model adjustment for gender, age, BMI, and sequence. These results indicate that walnut consumption for 16 weeks might affect glucose metabolism by decreasing $\mathrm{HbA} 1 \mathrm{c}$ and fasting blood glucose and by increasing circulating adiponectin.

\section{Plasma fatty acids profile}

Baseline fatty acid profiles before each intervention are summarized in Table 6 . None of the blood fatty acid profiles differed between the two intervention groups. However, there was a significant difference in myristic acid levels $(P=0.000)$ between walnut and control interventions (Table 6). Notably, alpha-linolenic acid (ALA) level after the walnut intervention was significantly increased over that after the control intervention $(P=0.012)$. The changes in ALA constitution is reflective of the fatty acid composition of walnuts and confirmed the self-reported changes in fatty acid intake. Walnut intervention did not affect other fatty acids such as arachidonic, eicosapentaenoic, docosapentaenoic and docosahexaenoic acids.

\section{DISCUSSION}

Our study results show that supplementing a subject's habitual diet with $45 \mathrm{~g}$ of walnuts daily for 16 weeks improved MetS status with $28.6 \%-52.8 \%$ reversion rates for individual MetS components. Consequently, 51.2\% of participants with MetS at baseline reverted back to normal status after the walnut intervention. Based on the results from linear mixed model adjustments for gender, age, BMl, and sequence, walnut consumption favorably changed fasting blood glucose, $\mathrm{HbA1c}$, HDL-C, adiponectin, and ALA in MetS subjects.

As stated in a health claim made by the U.S. Food and Drug Administration in 2004, among tree nuts, walnuts have been found to be particularly promising in improving blood lipid level [15]. Several intervention and cross-sectional studies have demonstrated that PUFA intake through walnuts can improve TC, LDL-C, and HDL-C levels [16-18]. Walnuts not only contain a high level of PUFAs, but also a low amount of saturated fatty acids and high amounts of dietary fiber, antioxidants, and phytosterols $[19,20]$. Plant phenols, which are richer in walnuts (1,625 mg gallic acid equivalents per $100 \mathrm{~g}$ of walnuts) than in other nuts, may possess various bioactive health benefits including the capacity to lower serum LDL cholesterol and/or promote detoxification. Thus, as a consequence, various actual health benefits might be observed. In particular, ALA in walnuts may work in synergy with other nutrients such as mono- and polyunsaturated fatty acids, proteins, and fiber [20]. Such components of walnuts can lead to a positive effect on various aspects of health. Cross-sectional and cohort studies have shown that nut consumption was related to a low risk of insulin secretion [21] and glucose homeostasis [22], which may explain the inverse association between habitual nut consumption and the risk of T2D. Moreover, a previous intervention study reported the consumption of $56 \mathrm{~g}$ of mixed nuts every day, as a replacement for carbohydrate food items, improved glycemic control in patients with T2D [23]. Similar to those previous results, our findings also show improved fasting blood glucose levels after walnut intervention compared with that after control food intervention. In addition, compared to our control results, there was a significant decrease in $\mathrm{HbA} 1 \mathrm{c}$ detected after the walnut intervention, in contrast to the results from a previous observational study [5]. Those authors reported that 
a $43 \mathrm{~g}$ daily walnut intake for eight weeks increased $\mathrm{HbA1c}$ among healthy subjects, whereas their blood lipid parameters improved. Subjects in both studies did not have severe diabetes and their $\mathrm{HbA} 1 \mathrm{c}$ levels were within the normal range before and after the walnut interventions. Further studies may be needed to investigate the effects of walnut intake on glucose metabolism among subjects with clinical T2D.

One of the major outcomes of the current study is that regular walnut intake among adults with MetS may have desirable effects, not only on fasting glucose and $\mathrm{HbA1c}$ but also on circulating adiponectin. The increased adiponectin level among the subjects with MetS might be evidence of a potential antidiabetic effect of walnuts. Adiponectin is an adipocyte-derived anti-atherogenic and anti-diabetic hormone and is abundant in human plasma [24]. Blood levels of adiponectin are negatively regulated by an increase in visceral fat [25] and therefore are lower in obese individuals [26]. Moreover, clinical studies showed hypoadiponectinemia in the pathogenesis of T2D [27], coronary artery disease [28], and hypertension [29]. Also, there have been previous reports showing a significant inverse relationship between adiponectin and insulin resistance. In particular, $\mathrm{HbA} 1 \mathrm{c}$ has been shown to have a negative correlation with serum adiponectin [30]. Hypoadiponectinemia seems to increase the risk of developing diabetes, and patients with MetS are at high risk of developing T2D [31]. Since the current study was performed with free-living subjects that added walnuts without other dietary controls or restriction, changes of adiponectin after walnut consumption for 16 weeks along with changes of glucose and $\mathrm{HbA} 1 \mathrm{c}$ might provide strong evidence for an anti-diabetic effect of walnuts. A previous study evaluated the effects of ALA on adiponectin in T2D patients and indicated that ALA supplementation can improve glucose homeostasis and is associated with an increase in adiponectin [32]. In healthy humans, SFA intake was correlated negatively with circulating adiponectin levels, whereas omega-3 PUFA was positively associated with circulating adiponectin levels [33]. In our study, after the walnut intervention, the subjects increased their intakes of PUFA $(P<0.0001)$ and ALA $(P<0.0001)$. In addition, blood ALA after walnut consumption was significantly increased. The data suggest that the intake of walnut on a regular basis may have a direct influence on plasma fatty acid composition. A previous crossover study also showed a significant increase in blood ALA after the study's walnut diet period [34]. Our results suggest that the increased ALA intake from walnut consumption was principally responsible for increasing the levels of adiponectin, even though the potential mechanisms for the effect are still not fully described. Further studies are needed to elucidate the changes in glucose and adiponectin metabolism induced by walnut consumption among subjects with clinical T2D.

Walnut consumption for 16 weeks also improved other parameters, despite the lack of detection of statistically significant intervention effects. Results from paired $t$-tests showed that blood levels of TG, TC, and apoB were reduced during the walnut intake period. Lipid-lowering effects of walnut have been investigated for decades; however, the mechanism by which walnuts reduce atherogenic lipoproteins is still unclear. As $\mathrm{Wu}$ et al. [34] pointed out, there are no available data to determine whether an improved lipid profile is a result of decreased production or increased clearance of apoB or a combination of both. Our results also showed an inverse relationship between walnut consumption and one of the atherogenic lipoprotein levels, although our results did not reveal a significant intervention effect.

Among the major strengths of this study is its randomized, crossover design with an addition of a washout period. The study systematically assessed the effect of walnut consumption on fasting lipid and glucose metabolism parameters, circulating adiponectin and leptin levels, as well as blood pressure and anthropometric measurements among free-living individuals. In addition, our study had an adequate sample size and appropriate intervention period. Our results have identified lipid-improving effects of a walnut dietary supplement in individuals with MetS. However, there is still a limitation to the study. Iso-caloric white bread, the control intervention, does not match the macronutrient content of walnuts due to its high percentage of carbohydrates. However, our results still showed that consuming walnuts as a substitute for a high carbohydrate snack (which is popular among Koreans) could be a healthy choice when managing MetS components.

In conclusion, our study shows that a daily dietary supplement of $45 \mathrm{~g}$ of walnuts for 16 weeks can favorably change MetS status by increasing the concentration of HDL cholesterol and decreasing the fasting glucose level. Furthermore, consuming walnuts on a daily basis also favorably changed $\mathrm{HbA} 1 \mathrm{c}$ and circulating adiponectin levels, which may explain its potential anti-diabetic effects.

\section{CONFLICT OF INTEREST}

The authors declare no potential conflicts of interests.

\section{ORCID}

Hyo-Jeong Hwang: https://orcid.org/0000-0003-2041-1934

Yanan Liu: https://orcid.org/0000-0002-6170-9414

Hyun-Sook Kim: https://orcid.org/0000-0002-1095-3660

Heeseung Lee: https://orcid.org/0000-0003-1238-9984

Yunsook Lim: https://orcid.org/0000-0002-3408-8595

Hyunjin Park: https://orcid.org/0000-0002-4869-0798

\section{REFERENCES}

1. Grundy SM, Hansen B, Smith SC Jr, Cleeman JI, Kahn RA; American Heart Association; National Heart, Lung, and Blood Institute; American Diabetes Association. Clinical management of metabolic syndrome: report of the American Heart Association/National Heart, Lung, and Blood Institute/American Diabetes Association conference on scientific issues related to management. Circulation 2004;109: 551-6.

2. Lim S, Shin H, Song JH, Kwak SH, Kang SM, Yoon JW, Choi SH, Cho SI, Park KS, Lee HK, Jang HC, Koh KK. Increasing prevalence of metabolic syndrome in Korea: the Korean National Health and Nutrition Examination Survey for 1998-2007. Diabetes Care 2011;34: 1323-8.

3. Statistics Korea. Annual report on the causes of death statistics 
[Internet]. Daejeon: Statistics Korea; 2014 [cited 2017 August 8]. Available from: http://kostat.go.kr/portal/korea.

4. Ministry of Health and Welfare, Korea Centers for Disease Control and Prevention. Korea Health Statistics 2014: Korea National Health and Nutrition Examination Survey (KNHANES VI-2). Cheongwon: Korea Centers for Disease Control and Prevention; 2014.

5. Bamberger C, Rossmeier A, Lechner K, Wu L, Waldmann E, Stark RG, Altenhofer J, Henze K, Parhofer KG. A walnut-enriched diet reduces lipids in healthy Caucasian subjects, independent of recommended macronutrient replacement and time point of consumption: a prospective, randomized, controlled trial. Nutrients 2017;9:9.

6. Rock CL, Flatt SW, Barkai HS, Pakiz B, Heath DD. Walnut consumption in a weight reduction intervention: effects on body weight, biological measures, blood pressure and satiety. Nutr J 2017;16:76.

7. Chisholm A, Mann J, Skeaff M, Frampton C, Sutherland W, Duncan A, Tiszavari S. A diet rich in walnuts favourably influences plasma fatty acid profile in moderately hyperlipidaemic subjects. Eur J Clin Nutr 1998;52:12-6.

8. Banel DK, Hu FB. Effects of walnut consumption on blood lipids and other cardiovascular risk factors: a meta-analysis and systematic review. Am J Clin Nutr 2009;90:56-63.

9. Aronis KN, Vamvini MT, Chamberland JP, Sweeney LL, Brennan AM, Magkos F, Mantzoros CS. Short-term walnut consumption increases circulating total adiponectin and apolipoprotein A concentrations, but does not affect markers of inflammation or vascular injury in obese humans with the metabolic syndrome: data from a double-blinded, randomized, placebo-controlled study. Metabolism 2012;61:577-82.

10. Katz DL, Davidhi A, Ma Y, Kavak Y, Bifulco L, Njike VY. Effects of walnuts on endothelial function in overweight adults with visceral obesity: a randomized, controlled, crossover trial. J Am Coll Nutr 2012;31:415-23.

11. Mukuddem-Petersen J, Stonehouse Oosthuizen W, Jerling JC, Hanekom SM, White Z. Effects of a high walnut and high cashew nut diet on selected markers of the metabolic syndrome: a controlled feeding trial. Br J Nutr 2007;97:1144-53.

12. Casas-Agustench $P$, López-Uriarte $P$, Bulló $M$, Ros E, Cabré-Vila JJ, Salas-Salvadó J. Effects of one serving of mixed nuts on serum lipids, insulin resistance and inflammatory markers in patients with the metabolic syndrome. Nutr Metab Cardiovasc Dis 2011;21:126-35.

13. Folch J, Lees $M$, Sloane Stanley GH. A simple method for the isolation and purification of total lipides from animal tissues. J Biol Chem 1957;226:497-509.

14. Morrison WR, Smith LM. Preparation of fatty acid methyl esters and dimethylacetals from lipids with boron fluoride-methanol. J Lipid Res 1964;5:600-8.

15. Food and Drug Administration, Office of Nutritional Products, Labeling, and Dietary Supplements. Qualified Health Claims: Letter of Enforcement Discretion-Walnuts and Coronary Heart Disease [Docket No. 02P-0292]. College Park (MD): Center for Food Safety and Applied Nutrition; 2004.

16. O'Neil CE, Fulgoni VL 3rd, Nicklas TA. Tree Nut consumption is associated with better adiposity measures and cardiovascular and metabolic syndrome health risk factors in U.S. Adults: NHANES 2005-2010. Nutr J 2015;14:64.

17. O'Neil CE, Keast DR, Nicklas TA, Fulgoni VL 3rd. Nut consumption is associated with decreased health risk factors for cardiovascular disease and metabolic syndrome in U.S. adults: NHANES 1999-2004. J Am Coll Nutr 2011;30:502-10.

18. Tapsell LC, Gillen L, Patch CS, Batterham M, Owen A, Baré M, Kennedy $M$. Including walnuts in a low-fat/modified-fat diet improves HDL cholesterol-to-total cholesterol ratios in patients with type 2 diabetes. Diabetes Care 2004;27:2777-83.

19. Ryan E, Galvin K, O'Connor TP, Maguire AR, O'Brien NM. Fatty acid profile, tocopherol, squalene and phytosterol content of brazil, pecan, pine, pistachio and cashew nuts. Int J Food Sci Nutr 2006;57:219-28.

20. Chen CY, Blumberg JB. Phytochemical composition of nuts. Asia Pac J Clin Nutr 2008;17 Suppl 1:329-32.

21. Estruch R, Martínez-González MA, Corella D, Salas-Salvadó J, Ruiz-Gutiérrez V, Covas Ml, Fiol M, Gómez-Gracia E, López-Sabater MC, Vinyoles E, Arós F, Conde M, Lahoz C, Lapetra J, Sáez G, Ros E; PREDIMED Study Investigators. Effects of a Mediterranean-style diet on cardiovascular risk factors: a randomized trial. Ann Intern Med 2006;145:1-11.

22. Jenkins DJ, Kendall CW, Marchie A, Josse AR, Nguyen TH, Faulkner DA, Lapsley KG, Singer W. Effect of almonds on insulin secretion and insulin resistance in nondiabetic hyperlipidemic subjects: a randomized controlled crossover trial. Metabolism 2008;57:882-7.

23. Jenkins DJ, Kendall CW, Banach MS, Srichaikul K, Vidgen E, Mitchell S, Parker T, Nishi S, Bashyam B, de Souza R, Ireland C, Josse RG. Nuts as a replacement for carbohydrates in the diabetic diet. Diabetes Care 2011;34:1706-11.

24. Iwaki M, Matsuda M, Maeda N, Funahashi T, Matsuzawa $Y$, Makishima M, Shimomura I. Induction of adiponectin, a fat-derived antidiabetic and antiatherogenic factor, by nuclear receptors. Diabetes 2003;52:1655-63.

25. Ryo $M$, Nakamura $T$, Kihara $S$, Kumada $M$, Shibazaki $S$, Takahashi M, Nagai M, Matsuzawa Y, Funahashi T. Adiponectin as a biomarker of the metabolic syndrome. Circ J 2004;68:975-81.

26. Arita Y, Kihara S, Ouchi N, Takahashi M, Maeda K, Miyagawa J, Hotta K, Shimomura I, Nakamura T, Miyaoka K, Kuriyama H, Nishida M, Yamashita S, Okubo K, Matsubara K, Muraguchi M, Ohmoto Y, Funahashi T, Matsuzawa Y. Paradoxical decrease of an adiposespecific protein, adiponectin, in obesity. 1999. Biochem Biophys Res Commun 2012;425:560-4.

27. Spranger J, Kroke A, Möhlig M, Bergmann MM, Ristow M, Boeing $\mathrm{H}$, Pfeiffer AF. Adiponectin and protection against type 2 diabetes mellitus. Lancet 2003;361:226-8.

28. Laughlin GA, Barrett-Connor E, May S, Langenberg C. Association of adiponectin with coronary heart disease and mortality: the Rancho Bernardo study. Am J Epidemiol 2007;165:164-74.

29. Iwashima $Y$, Katsuya T, Ishikawa $K$, Ouchi N, Ohishi M, Sugimoto K, Fu Y, Motone M, Yamamoto K, Matsuo A, Ohashi K, Kihara S, Funahashi T, Rakugi H, Matsuzawa $Y$, Ogihara T. Hypoadiponectinemia is an independent risk factor for hypertension. Hypertension 2004;43:1318-23.

30. Goodarzi MT, Babaahmadi-Rezaei $H$, Kadkhodaei-Eliaderani $M$, Haddadinezhad S. Relationship of serum adiponectin with blood lipids, $\mathrm{HbA}_{1} \mathrm{c}$, and hs-CRP in type II diabetic postmenopausal women. J Clin Lab Anal 2007;21:197-200.

31. Esfahani M, Movahedian A, Baranchi M, Goodarzi MT. Adiponectin: an adipokine with protective features against metabolic syndrome. Iran J Basic Med Sci 2015;18:430-42. 
32. Gomes PM, Hollanda-Miranda WR, Beraldo RA, Castro AV, Geloneze B, Foss MC, Foss-Freitas MC. Supplementation of a-linolenic acid improves serum adiponectin levels and insulin sensitivity in patients with type 2 diabetes. Nutrition 2015;31:853-7.

33. Fernández-Real JM, Vendrell J, Ricart W. Circulating adiponectin and plasma fatty acid profile. Clin Chem 2005;51:603-9.
34. Wu L, Piotrowski K, Rau T, Waldmann E, Broedl UC, Demmelmair H, Koletzko B, Stark RG, Nagel JM, Mantzoros CS, Parhofer KG. Walnut-enriched diet reduces fasting non-HDL-cholesterol and apolipoprotein B in healthy Caucasian subjects: a randomized controlled cross-over clinical trial. Metabolism 2014;63:382-91. 